Please do not remove this page

RMIT

UNIVERSITY

\title{
'What if you live on top of a bakery and you like cakes?'-Drug use and harm trajectories before, during and after the emergence of Silk Road
}

Barratt, Monica; Lenton, Simon; Maddox, Alexia; Allen, Matthew

https://researchrepository.rmit.edu.au/esploro/outputs/9921861098201341/filesAndLinks?institution=61RMIT_INST\&index=null

Barratt, M., Lenton, S., Maddox, A., \& Allen, M. (2016). "What if you live on top of a bakery and you like cakes?"-Drug use and harm trajectories before, during and after the emergence of Silk Road. International Journal of Drug Policy, 35, 50-57. https://doi.org/10.1016/j.drugpo.2016.04.006 Document Version: Accepted Manuscript

Published Version: https://doi.org/10.1016/j.drugpo.2016.04.006

Repository homepage: https://researchrepository.rmit.edu.au

CC BY-NC-ND V4.0

(C) 2016 Elsevier B.V. All rights reserved.

Downloaded On 2023/04/26 19:47:09 +1000 
'What if you live on top of a bakery and you like cakes?' - Drug use and harm trajectories before, during and after the emergence of Silk Road

Monica J. Barratt ${ }^{\mathrm{a}, \mathrm{b}, \mathrm{c}}$, Simon Lenton ${ }^{\mathrm{b}}$, Alexia Maddox ${ }^{\mathrm{b}}$, Matthew Allen ${ }^{\mathrm{d}}$

${ }^{a}$ Drug Policy Modelling Program, National Drug and Alcohol Research Centre, UNSW

Australia, Sydney NSW 2052, Australia. Email: m.barratt@unsw.edu.au

${ }^{\mathrm{b}}$ National Drug Research Institute, Faculty of Health Sciences, Curtin University, GPO Box

U1987, Perth WA 6845, Australia. Email: s.lenton@curtin.edu.au; a.maddox@deakin.edu.au

${ }^{\mathrm{c}}$ Centre of Population Health, Burnet Institute, 85 Commercial Road, Melbourne Victoria 3004, Australia.

d School of Communication and Creative Arts, Deakin University, 1 Gheringhap Road Geelong, Victoria, Australia. Email: matthew.allen@deakin.edu.au

Corresponding author:

Monica J. Barratt

Drug Policy Modelling Program

National Drug and Alcohol Research Centre

UNSW Australia, Sydney NSW 2052, Australia

Email: m.barratt@unsw.edu.au

Note: This paper was presented at the $9^{\text {th }}$ meeting of the International Society for the Study of Drug Policy, Ghent, Belgium, 20-22 May 2015. 
Abstract

Background: Cryptomarkets are digital platforms that use anonymising software (e.g. Tor) and cryptocurrencies (e.g. Bitcoin) to facilitate peer-to-peer (P2P) trade of goods and services. Their emergence has facilitated access to a wide range of high-quality psychoactive substances, according to surveys of users. In this paper, we ask the question 'How does changing access to drugs through cryptomarkets affect the drug use and harm trajectories of their users?'

Method: We conducted a digital ethnography spanning 2012-2014, a period that included the seizure of the original Silk Road marketplace and forum by law enforcement. Using encrypted online chat, we interviewed 17 people who reported using Silk Road to purchase illicit drugs. The interviews were in-depth and unstructured, and also involved the use of life history timelines to trace trajectories. Transcripts were analysed thematically using NVivo.

Results: For some, Silk Road facilitated initiation into drug use or a return to drug use after cessation. Typically, participants reported experiencing a glut of drug consumption in their first months using Silk Road, described by one participant as akin to 'kids in a candy store'. There was evidence that very high availability reduced the need for drug hoarding which helped some respondents to moderate use and feel more in control of purchases made online.

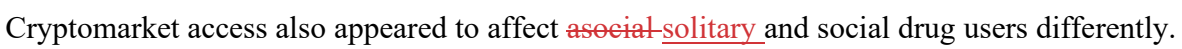
Most participants described using other cryptomarkets after the closure of Silk Road, albeit with less confidence.

Discussion: In the context of high levels of drug access, supply and diversity occurring within a community regulated environment online, the impacts of cryptomarkets upon drug use trajectories are complex, often posing new challenges for self-control, yet not always leading to harmful outcomes. A major policy challenge is how to provide support for harm reduction in these highly volatile settings.

Keywords (3-6)

Cryptomarkets, drug markets, availability, drug trajectories, digital ethnography, Silk Road

Word count $=7011 \underline{7188}$ 


\section{Introduction}

Cryptomarkets are digital platforms that use anonymising software (e.g. Tor) and cryptocurrencies (e.g. Bitcoin) to facilitate peer-to-peer (P2P) trade of goods and services.

Their emergence has facilitated access to a wide range of psychoactive substances, according to website-marketplace analyses (Aldridge \& Décary-Hétu, 2014; Christin, 2013; Martin, 2014) using digital trace methodology -(Décary-Hétu \& Aldridge, 2015) and surveys of users (Barratt, Ferris, \& Winstock, 2014). As they have increased in popularity and visibility, it has become more important to understand how drug-using and drug-buying practices may have evolved in response to cryptomarkets. Without knowing more about how people who buy drugs from cryptomarkets are affected, we cannot fully assess the potential harms and/or benefits of cryptomarkets. The interviewee quotation forming the title of this paper-"What if you live on top of a bakery and you like cakes?"-leads us to question how the specific nature of drug access associated with the emergence of cryptomarkets may prompt different kinds of relationships towards drugs that are now more easily available.

According to Martin, cryptomarkets are "online forum(s) where goods and services are exchanged between parties who use digital encryption to conceal their identities", distinguishable from other websitesdrug vendors operating in the 'clear' or 'surface' web through their use of: the Tor network (see below), third party hosting, the postal service to deliver goods, vendor and buyer rating systems, decentralised exchange networks, and cryptocurrencies (Martin, 2014, pp. 2-3). They are hosted in the dark net or dark web, a part of the internet that is not indexed by conventional search engines and is only accessed by specialised browsers. In contrast, the clear or surface web may be defined as all content accessible via search engines using a normal browser. For example, .onionCryptomarkets and other sites in the dark net sites-must be accessed using Tor or similar browsers. This anonymization softwareusing the Tor (The Onion Router) browser: software that makes it mere-very difficult to track the identities and locations of network users (Dingledine,

Mathewson, \& Syverson, 2004). Eryptomarkets may also use other anonymising networks, for example, I2P (The Invisible Internet Project), although Tor is currently the most commonly used.Silk Road, the original cryptomarket that ran from 2011 to 2013, not only operated as a place to trade drugs, but through its associated discussion forums, an active online community formed where the often stigmatised topic of drug use was openly discussed and the values of cyber-libertarianism were espoused (Maddox, Barratt, Allen, \& Lenton, 2016). 
A review of the literature surrounding the effect of changes in drug availability upon the trajectories of drug use and harm is valuable for assessing the cryptomarket phenomenon from a public health perspective. The influence of availability of substances upon population prevalence of use and harm has been a topic of much scholarship, particularly among epidemiologists studying alcohol (Liang \& Chikritzhs, 2011; Livingston, 2014; Stockwell \& Gruenewald, 2004) and increasingly the supply of cannabis through medical marijuana dispensaries (Freisthler \& Gruenewald, 2014). Understanding the consequences of sudden reductions in drug availability, for example, after law enforcement efforts result in market disruption or displacement, is the goal of the 'hot-spot' literature (Kerr, Small, \& Wood, 2005). Furthermore, analysts concerned with modelling changes to drug policies, for example, the evaluation of cannabis policy reforms involving moving to a decriminalised or regulated supply model, have discussed theories of availability and its relationship to prevalence (Shanahan \& Ritter, 2014)

While area-level analyses demonstrate that increased density of outlets (physical availability) predicts increased substance use and related harms (e.g., alcohol-related assaults, Livingston, 2008) after statistically controlling for a variety of confounding factors, it is not clear what the mechanisms of action actually are (Gruenewald, 2008; Livingston, 2013). The relationship between availability and subsequent substance related harm is complex and multifaceted. Another important consideration is that associations between variables at the area level do not necessarily predict associations at the individual level (the ecological fallacy). Indeed, this methodological problem has led some to argue that "[p]urely aggregatelevel studies examining total outlet density only should be abandoned" (Gmel, Holmes, \& Studer, 2015 Early View). While we are also interested in the implications of cryptomarkets at the population level, this paper is primarily concerned with describing and understanding the diversity of drug trajectories of individuals exposed to situations of sudden high availability of a wider variety of better-quality illicit drugs. Such individual diversity may be cancelled out in area-level studies.

Abbey et al. (1993) found that levels of social and subjective availability of alcohol (measured at the individual level) predicted alcohol consumption whereas physical availability (measured at the local level) did not. They explain the concept of subjective availability using this example:

[A]n urban resident may live very close to an alcohol outlet, but be afraid to walk through her neighborhood alone. In this case, alcohol is physically but not subjectively 
available. In contrast, a rural resident may live a long distance from an alcohol outlet, but the distance he has to travel to obtain alcohol may be no greater than the distance he has to travel to obtain groceries and gasoline. In this case, alcohol is subjectively but not physically available" (p. 490).

It was also noted by Babor and colleagues, in their recognition of the importance of availability for understanding patterns of drug use, that it refers "not only to the supply of drugs (physical availability), but also to their cost (economic availability), their attractiveness (psychological availability), and their social acceptance within the user's primary reference groups (social availability)" (Babor et al., 2010, p. 18).

Applying these types of availability to cryptomarkets, we may posit that those who have the necessary technical skills and cultural understandings to operate successfully in the dark net setting - that is, they have set up a system that is easy for them and that they trust—will experience higher levels of subjective availability, compared with cryptomarket users who are unfamiliar with the environment. Although it may appear that both people-technically familiar and unfamiliar populations with the same access to information technology and

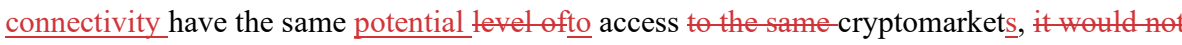
be perceived as easily obtainable in the same waytheir perceptions of ease of availability may differ. Furthermore, for those who live in areas where drugs are relatively more expensive to access in person, use of cryptomarkets may increase economic as well as physical availability, even more so if the cryptomarket user buys in bulk and sells enough to cover their own usage costs. Social availability is also important, because for those people who live within social and cultural milieux where drug use is not generally acceptable, access to and connection with the online communities associated with cryptomarkets may increase the social availability and acceptance of their use within that setting.

In this paper we are interested in understanding how the emergence of the first cryptomarket, Silk Road, affected the drug use and harm trajectories of its users. Using qualitative methods, we describe these trajectories before, during and after its emergence.

\section{Methodology}

\section{Approach}

In contrast to the bulk of research conducted into cryptomarkets that employs digital trace analyses (e.g., Aldridge \& Décary-Hétu, 2014; 2016; Christin, 2013; Dolliver, 2015; Phelps 
$\&$ Watt, 2014), we took an active participatory approach to researching the experiences of illicit drug buyers on Silk Road. A similar approach has been taken by van Hout and Bingham (2013a, 2013b, 2014), who conducted their qualitative studies of Silk Road participants prior to the marketplace's closure. Our study can be described as a multi-sited ethnography, an approach which recognises that objects of inquiry, such as people, things, information and ideas, are networked and dispersed across time and space (Falzon, 2009; Hine, 2008). As an ethnography, our study seeks to explain practices that appear 'irrational' from one perspective by providing rich description of the cultural logics of informants, to whom the practice makes sense (Agar, 2006). It was multi-sited in the sense that we did not focus on a singular field-site (e.g. the Silk Road marketplace and forums). Instead, we traced the movements of populations that were (digitally) dispersed after the FBI seizure of Silk Road, which occurred just as we were about to launch our study through the Silk Road forums.

\section{Procedure}

Familiarisation with the dark web and the Silk Road digital space was ongoing from our discovery of the site in 2011 (see Barratt, 2012a). Our study preparation included the development of a security protocol to ensure anonymity of our participants, practice and mastery of these technical skills, and amendments to our methodology to match changes in the dynamic dark web setting. During the period of entering the field, we focused on the development of a credible researcher identity within the community and monitoring community dynamics in response to our presence. Van Hout and Bingham (2013a, p. 386) note in their first attempt to access the Silk Road community: "recruitment of site users was hampered by negative and suspicious reactions by forum participants". Community responses to our call for participation in the forum recruitment thread included a mix of negative and positive reactions. By the continued engagement that was required by actively recruiting participants in the interlinked online locales of Twitter, Reddit and cryptomarket forums and chat channels, we learned much about the tensions within these networks between keeping the markets open to new entrants or remaining closed for greater protection from law enforcement or other outsiders, including researchers. In addition to the interviews, we contributed to and archived some community discussions occurring in forums and chat channels, and conducted member checking exercises with homologous communities, for example, at the local Bitcoin meetups -(see Barratt \& Maddox, forthcoming In press, for greater detail on our ethnographic methods). 
The data reported in this paper are derived solely from A.M. conducted 17 encrypted online chat interviews conducted by A.M., which involved synchronous or 'real-time' textual exchange, requiring co-presence of the interviewer and interviewee (see Barratt, 2012b). The interviews were conducted between September 2013 to June 2014, with all but one interview conducted after the closure of Silk Road. Participants were required to be aged 18 years or over and to report buying drugs at least once from Silk Road. Interviews took 3 hours to complete on average, ranging from 2 to 4 hours. No monetary compensation was offered. A participant information sheet was available from the project website, and the interviewer (A.M.) obtained text-based consent to participate after provision of the link to this document and some short summaries of its contents, including reminders that participation was voluntary and that they could choose to stop the interview at any time. An encrypted channel was formed between A.M. and the interviewee, and the interviewee was also able to verify A.M.'s identity by matching her public key, also published on the project website, with her cryptographic fingerprint in the interview. In terms of interview style and content, the interview was unstructured. A.M. used a series of prompts to guide the conversation through topics of interest, which included a description of their engagement with Silk Road from their first discovery of it until its closure, their drug use trajectories, their computer and internet use histories, and how they dealt with the Silk Road closure and their experience with other cryptomarkets. Throughout this process, A.M. jotted information onto a printed life timeline template, and used this document to prompt further probing of the nature and dependencies of events in the interviewee's life.

After each interview was complete, A.M. de-identified the text log before saving the data. A participant I.D.identification number was assigned. Interview transcripts were entered into NVivo. During first pass coding, A.M. conducted open coding of content into in vivo nodes under predetermined node trees. A second pass through each interview transcript was completed to apply these new nodes more consistently. To assist with validity and transparency of coding, M.B. selected 2 transcripts using a random number generator to code using the same node structure. We calculated kappa values to test for coding similarity and divergence between coders for these two example cases. The kappa values allowed us to more efficiently and systemically identify nodes where further discussion was necessary to refine the definition and scope of the theme. According to McHugh (2012), kappa values of 0.6 or above indicate at least moderate agreement between coders. One quarter $(28 \%)$ of nodes had kappa values below 0.6 , and were therefore subjected to further scrutiny where 
discrepancies between coders were resolved. Following this process, A.M. conducted a final pass over the dataset applying the coding structure assisted by refined code definitions.

\section{Analysis}

Each transcript was read as a whole alongside the corresponding life timeline. For each transcript, M.B. distilled the self-reported drug use trajectories into categories of drug use patterns and noted at which point in these trajectories cryptomarket drug buying was initiated or discontinued. For each analytic section, these trajectories were arranged into thematic categories based on the nature of the changes in drug use patterns. To support the analysis, text coded to relevant nodes were drawn upon when seeking examples and counter-examples of emergent themes. To preserve the tone of the online chat interviews, texts are reproduced verbatim: that is, with their original spelling and typography. Additional explanatory information is provided within brackets.

\section{Ethics}

Our participant information sheet informed participants that they should refrain from providing any detailed information about criminal activities such as names and places. The study was approved by the Curtin Human Research Ethics Committee (HR 26/2012). Further analysis of the methodological and ethical issues arising from this study may be found at Barratt and Maddox (Forthcoming $\underline{\underline{I n} \text { press) }}$ ) and by contacting the authors to obtain the technical report.

\section{Results}

\section{Description of participants}

The median age of the 17 participants was 21-25 years (3 reported 18-20, 7 reported 21-25, 2 reported 31-35, 2 were over 35, 3 missing), and 15 participants identified as male (also 1 as 'non-binary gender', 1 missing). Of the 11 participants who identified their country of residence, 5 were from the USA, 4 from Australia, 1 from the UK and 1 from Sweden. Of the 14 that identified a geographical context, 8 specified living in a major city and 6 in a regional area. Of the 13 participants that described their educational attainment, 3 described only partial completion of secondary school, 4 reported completion of secondary school and 6 reported engagement in or completion of tertiary education. Of the 14 participants who described their occupation, 10 described their usual field of employment, 3 were students and 1 (aged over 50) was retired. Of the 10 describing employment, 4 worked in the technology 
sector, 4 in the retail sector, 1 in education and 1 in the media.

\section{Before Silk Road}

Some participants reported that Silk Road was the absolute start of their drug-using activities. P12 (21-25 yrs, UK) used Silk Road to purchase only LSD and cannabis over 14 months of Silk Road use. He reported that his first purchase from Silk Road of LSD was the first illegal drug he had ever used. He described that he "never had connections to buy drugs in real life, and I still don't, so SR was literally my only means of getting drugs, bar 'legal highs', and all that other crap on non-deepweb". Others had used only very occasionally prior to Silk Road access. P11 (21-25 yrs, Sweden) had also never bought drugs in-person or face-to-face, with the only drug experience he had prior to Silk Road was a one-off use of synthetic cannabis bought through the 'clear web'. ${ }^{+}$When asked why he moved online to buy cannabis and hashish, he responded "I didn't move online. I was always online, I've never purchased drugs in person. I don't like the idea of it." Similarly, P14 (21-25 yrs, USA) reported trying alcohol and cannabis obtained in-person briefly but not enjoying them, before using LSD bought from Silk Road, which he described as his "first recreational drug".

For others, the period before Silk Road use contained intensive drug use episodes, yet some themes around availability intersect with participants who were naïve to drugs before Silk Road. For example, P16 (missing age and country) described engaging in daily cannabis use in his youth after which he quit drugs for many years. His entry back into drug use occurred through the use of clear web vendors for research chemicals and cannabis. Similarly to P12 and P11 above, he stated 'I never look for drugs in real life. I don't have 'a dealer' or anything." P18 (36-40 yrs, Australia) described losing his real world drug dealing contacts due to age and circumstances, and finding himself "ringing around some friends and friends of friends, having to score by driving to a park at lam... I thought 'i'm too old for this shit"”. For P23 (18-20, Australia), working as a FIFO (fly in fly out) in a regional location meant that there was no access to drug supply and also meant that he was away from his support

\footnotetext{
${ }^{+}$The clear web, also known as the surface web, is all web content that is accessible via search engines and only requires a normal browser to access. In contrast, the deep web is web content that is inaccessible via search engines. A subset of the deep web is known by its users as the dark web, which includes hidden internet services accessed only through anonymising browsers (Tor, $\mathrm{I} 2 \mathrm{P}$ ).
} 
networks, which he attributed to his use of drugs (sourced through Silk Road) "for escapism". The remainder and bulk of the sample described obtaining drugs through conventional sources like friends and dealers prior to accessing Silk Road. Seven participants also described the use of clear web online stores prior to accessing Silk Road. Most of these online purchases from clear web stores were for new psychoactive substances, including Salvia divinorum, synthetic cannabinoid receptor agonists, and 'research chemicals', thus named by companies in an attempt to circumvent legislation prohibited their sale for human consumption (Sanders, Lankenau, Bloom, \& Hathazi, 2008). One respondent described using online pharmacies to purchase prescription drugs for recreational use, and another described purchasing cannabis from an online store (in the clear web).

\section{The honeymoon period}

The period directly after the first successful purchase of drugs from Silk Road could be described as a 'honeymoon period'. P4 (21-25 yrs, Australia) described how he and his friends felt once they had successfully purchased from Silk Road:

11:18:55 am P4: We no longer needed a dealer

11:19:11 am P4: We essentially knew the quality would be good based on reviews

11:19:29 am P4: watever we had the cash for and felt like, we could have it next business day 11:19:36 am P4: kids in a candy store

Most (14) participants' drug trajectories immediately after first use of Silk Road could be described as 'increasing', with 11 of those describing a tapering down of use after a honeymoon period, and 3 remaining at a stable increased pattern. A typical trajectory in these data included trying many new drug types for the first time. For example, P5 (18-20 yrs, country missing) described his drug use as "[A]ccelerated. Considering for 3 years I did 2 drugs or so and then at 16 [after Silk Road] I tried like 20 more”. Psychedelic drugs, including LSD, DMT, mushrooms and relatively new substances including from the NBOMe and $2 \mathrm{C}$ family, were most popular, as well as MDMA. For example, P20 (missing age, USA) stated "I've actually never bought LSD in person, only through SR" and P21 (missing age and country) said "Ever since i had heard about LSD, i wanted some immediately, so i went to the LSD section [on Silk Road] and found the people with the best price and reputation and ordered 4 tabs". There was another group of participants who also included drugs like heroin, cocaine and methamphetamine as drugs first tried through Silk Road. Most respondents also described purchasing cannabis through Silk Road, some for the first time. A minority 
purchased pharmaceutical drugs through Silk Road, some for the first time and others to supplement their medical supply.

\section{From scarcity to abundance}

Although it was typical among this sample for use to increase upon access to Silk Road, it was clear that for some participants, the capacity to more easily satiate desires for drugs necessitated new practices of self-control. Whereas before, for many, accessing drugs was either impossible or prohibitively difficult, Silk Road heralded a new state of abundance. This abundance of drug opportunities had an interesting effect for some of the participants: it was as if very high levels of availability resulted in a satiation of desire following initial intensive use. P1 (21-25 yrs, USA) explained that "[e]xcept for some meth I did in Amsterdam, I have only taken drugs that I have personally acquired through the sr since finding the silk road" and that "this whole summer, I've had such abundance. I really have no desire to take anything anymore." For P1, Silk Road was a trigger for his drug use to increase dramatically, peak then decline rapidly after an intensive period of drug use facilitated by very high availability. Similarly, P15 (21-25 yrs, USA) related the "ease of making an occasional order on SR as a treat" to his "decreased desire to do hard drugs" (which, for him, includes heroin and methamphetamine). Earlier in the interview, he provided an example of how he purchased $3.5 \mathrm{~g}$ of methamphetamine from SR, it arrived overweight at $5 \mathrm{~g}$, so he sold $1.5 \mathrm{~g}$ to a friend to reduce the temptation to use more than he had planned. P15 noted that "obviously it's easier to control yourself when you can't get it locally", indicating that he preferred being able to order in small amounts as needed to aid self-controlled use. This concept of 'portion control' was also described by P16 (missing age and country) using the bakery analogy:

[P16] what if you live on top of a bakery and you like cakes?

[P16] for a couple of weeks maybe you gain weight

[P16] maybe i'm there

[P16] i just don't want to develop diabetes

[P16] i can buy a slice when I want it

$[\mathrm{P} 16]$ not a slice of every cake and put them in my fridge.

[P16] or else

[P16] stay glued on the window of the bakery all day

P16 said that the key way of limiting his consumption was to limit his Tor browsing or "window shopping". He explained that "I tend to have an addictive personality but I try to 
limit myself”. Similarly, P21 (missing age and country) commented on his methamphetamine use, "i control it by not ordering it". He explained "i'm not addicted to the point where i'm going to need it all the time, but if i have it, i'm going to use it, pretty much". Yet, it was also apparent that while Silk Road increased access to all drug types, it was not uncommon for participants to delineate between acceptable and unacceptable drugs to use regardless of their availability. For example, P1 stated "I like the fact that I can get anything I want with relative ease. I have no desire to start taking xanax or heroin. If I have time, I'll probably buy some lsd. That's about it." P20 (missing age, USA) similarly stated "i never get into stuff like heroin or cocaine, so i'd say my use is pretty tame compared to some" and P24 (31-35 yrs, missing country) claimed "I bought nearly everything besides heroin, i draw the line at needles and crack."

Tapering down drug use after a honeymoon period was not only triggered by the practice of 'portion control' and/or satiated desires, but also by other life events. For example, P1 (21-25 yrs, USA) explained "After last semester I'm more interested in grades than highs" and P4 (21-25 yrs, Australia) described how "I'd rather buy a flight than a gram of coke". In the case of P25 (21-25 yrs, Australia), who was buying large amounts from Silk Road and dealing from his home, a police raid resulted in supply charges, which led to a tapering of drug use and cryptomarket activity.

\section{Complex changes in social availability}

A distinct theme in these data emerged regarding the perceived advantages of being able to avoid the social aspects of acquiring drugs in-person. For example, P20 (missing age, USA) stated "the benefits is that it's more cheap, i don't have to go through some sketchy dude in person". It became apparent that many (9) of the participants were asociatsolitary drug users, in that their drug use occurred alone, and without the knowledge of other people who knew them 'in real life'. Yet, most of these asocial-solitary drug users described a high level of engagement with the Silk Road online community - that is, they were not broadly asocial but rather, they kept their drug use private and separate from their in-person social networks. For example, P21 (missing age and country) stated "yeah i keep most of my drug use to myself irl [in real life], but i'm pretty open about it in the chat and on the forums". Similarly, P12 (21$25 \mathrm{yrs}, \mathrm{UK})$ noted "I never was involved in the kind of social circle that did drugs, and obviously given the VERY heavy social stigma behind drug use, it was never something I felt comfortable trying to share with anyone else... I've always enjoyed being able to share my 
experiences online with other drug users." Others expressed a preference for solo use. For example, P15 (21-25 yrs, USA) said that social use "dulled the experience. I like my psychedelics by myself." In contrast P11 (21-25 yrs, Sweden) "would LIKE to use it [cannabis] socially, if it were more socially acceptable."

Regarding engagement with the online community of drug users, this was not universally desired. P17 (31-35 yrs, missing country) stated "It is my vice, I like to handle it on my own". He ponders that perhaps other people want to be more social, but that he "never posted on the original forums. I am happy with a vendor who will give me no more contact than a box in the mail". P18 (36-40 yrs, Australia) described the possibility of solo drug use and the removal of the social aspect of access as "one drawback is that it can make drug using quite an insular activity if you're in my situation, which it never used to be". It may be the case that these asocial solitary drug users would either reluctantly seek illicit drugs through in-person social networks, or would not use illicit drugs at all if the cryptomarket opportunities were not available.

Other participants described social drug taking practices, most of which involved social supply or minimally commercial supply (Coomber \& Moyle, 2014). Typically, the participants in our study began sharing drugs they bought through Silk Road with their friends which led to them buying on behalf of friends. For example, P21 (missing age and country) recalled that "after a few group purchases with friends, people started making orders through me... eventually, i would buy more than i needed of something i'd want to try and then sell it to my friends. so my drugs were essentially free or i was getting paid to do them". Although we specifically did not ask participants to describe drug selling, it was apparent in the data that the opportunity to buy in large quantities and on-sell to friends was a key aspect that fuelled drug consumption among these social drug users, as it enabled the participant to buy more drugs with the profits made through sales. Some of them appeared to unintentionally fall into the role of supplier, while others described seeing the (financial) opportunity and intentionally pursuing it. For example, P5 (18-20 yrs, missing country) noted "It was easy to sell using SR and I fell right into it. It was so cheap and people paid so much", whereas P20 (missing age, USA) commented "I also saw the possible profit incentive in getting bulk marijuana mailed" and P23 (18-20, Australia), who described himself as a wholesaler, noted that he "realised the huge market potential of mdma in my city". Even among these participants, who described experiencing the Silk Road honeymoon intensively through funding increased drug use from dealing, tapering of drug use occurred due to 
insights obtained from psychedelics (P23) and reduced interest (P5).

\section{Atypical drug trajectories}

Three participants' drug use trajectories could be better described as stable or decreasing: access to Silk Road did not appear to affect the volume or variety of drugs used. In the case of P4 (21-25 yrs, Australia), he described some intense and harmful drug episodes prior to Silk Road, including an addictive period with methamphetamine where he amassed a large debt to his dealer. He began using Silk Road only one month before it was seized, and during this time, bought Duromine for the purposes of weight loss. P4 was also becoming more interested in travel than in drug use, so his trajectory was stable or decreasing. P15 (21-25 yrs, USA) also described bouts of dependent use pre Silk Road of methamphetamine and heroin. Although Silk Road heralded his use of a wide variety of substances, he describes his use of DMT (sourced through Silk Road) as a breakthrough experience that changed his outlook, resulting in a lessened desire to use "hard drugs". In the case of P24 (31-35 yrs, missing country), he had already been using the Farmers Market ${ }^{2}$ and private email lists to access illicit drugs online. When Silk Road opened, he described simply following the Farmers Market vendors onto Silk Road and continuing his drug buying practices using the new platform. Thus, unlike most Silk Road entrants in our study, P24 was not faced with a drastic change in availability, rather just a change in the platform for purchase.

\section{Trajectories of harm (and benefit)}

For some participants, the honeymoon period associated with the discovery and first use of Silk Road was also associated with increased drug-related harm. P23 (18-20 yrs, Australia) described Silk Road as "very dangerous" because at the time that he first began using it, "I really took anything". After taking liquid Xanax, P23 described becoming addicted and experiencing serious withdrawal symptoms. Methamphetamine use resulting in dependence, psychosis and exhaustion after bingeing was described by a handful of participants; for example, P5 (18-20 yrs, missing country) recalled that "The last time i was on meth I felt like i was dying for 2-3 days, it was wednesday before my body stopped aching, and I was still

\footnotetext{
${ }^{2}$ The Farmers Market utilised the dark web but was not using cryptocurrencies nor was it using an escrow or centralised digital platform to facilitate comparison of vendor ratings.
} 
exhausted". Other participants described performing poorly on exams due to excessive partying fuelled by drugs sourced through Silk Road. Nevertheless, participants reported benefits that they associated with their drug use that was facilitated by Silk Road, most notably the enjoyment and insightful experiences arising from psychedelic drugs not otherwise accessible, like DMT and LSD. P1 (21-25 yrs, USA) described the preceding year of his life, in which he accessed psychedelics from the Silk Road, as "one of the best developmental years for me in terms of growth and new positive relationships". P24 (31-35 yrs, missing country) showed A.M. his psychedelic-inspired artwork during the online interview as evidence of the creative benefits of his drug use. Beneficial and pleasurable experiences were also described following the use of a range of other drugs, including MDMA, cannabis, ketamine, GHB, methamphetamine and opiates. Still other participants did not identify benefits but resisted the idea of harms arising from their use patterns. For example, P19 (18-20 yrs, USA) reported the use of heroin by insufflation (nasal inhalation) once or twice a month "so I'm a far cry from getting addicted. Not many people can have this level of self-control, I concede, but I've been doing this for more than a year now and it has not harmed any aspect of my life." Participants also identified experiencing harms from their drug use that were related to the cultural and legal context of illicit drugs rather than their effects in the body, including stigma, feeling that they have to lie about their use to family and friends to avoid detection, and legal problems.

\section{After Silk Road}

After the FBI seizure of Silk Road in October 2013, almost all of the participants in this study described using alternative cryptomarkets (11 used other cryptomarkets, 2 had not yet done so, 3 missing, 1 not applicable as interview occurred before closure). Generally, though, their level of comfort using cryptomarkets to buy drugs was reduced, as the market changed from one trusted player to multiple players of unknown trustworthiness. The use of direct dealing, where buyers use encrypted email or chat to arrange purchases with vendors they had previously dealt with through cryptomarkets, was reported by 3 participants after the Silk Road closure. Some participants continued to purchase through the dark net but with increased security protocols, such as only using public WiFi hotspots (to reduce the chance of being traced from vulnerability in Tor) or avoiding international transactions (to reduce the chance of being caught through customs' inspection of packages).

The sense of abundance, and the complex changes in purchasing patterns that this sense can 
lead to, may be undermined by sudden market closures due to law enforcement or scams. P16 (missing age and country) noted "If they continue to close more and more markets, people could develop the syndrome of compulsive buying or buying 'to stash up'. Just in case online drug market disappear." For some participants in this study, feeling the need to buy in bulk due to anxiety about continuous availability into the future may make it harder to control their use. As P16 summarised "[t]his is no harm reduction".

\section{Discussion}

From the perspective of epidemiological area-level studies, it is generally accepted that increased availability of drugs is associated with increased prevalence of use and subsequent harm. Our study, consistent with Abbey et al. (1993) and Babor et al (2010), indicates that there is a more complex story to tell about how changes in drug availability affect drug use and harm trajectories. While it was unsurprising to find that our respondents typically increased frequency of use and tried new types of drugs during the 'honeymoon' period, there was evidence that consistently high availability reduced the need for purchasing larger quantities of drugs (e.g. buying the whole cake). This reduction in hoarding behaviours helped some respondents to moderate their use and feel more in control of purchases made online (e.g. buying one piece of cake at a time). There was also evidence that the desire for drugs can become satiated after the honeymoon period triggered by the saturation availability of a previously scarce drug. For some, high availability may assist them in maintaining a controlled drug use pattern resulting in reduced harm, whereas for others, high availability facilitated intense use and subsequent harm. Nevertheless, respondents typically reported a reduction or tapering of use following the honeymoon period, in the absence of any specific intervention. Additionally, while some drug use harms were reported, specifically related to excessive methamphetamine and benzodiazepine use, other drug use episodes were described simply pleasurable or as instrumental in reducing overall drug-related harm, most notably psychedelic use that led to personal insights and positive behavioural change. These findings suggest that the mere availability of cryptomarkets for illicit drug transactions does not determine an increased use or increased harm.

Our research demonstrates the importance of considering different kinds of drug availability: physical, social, and economic (Abbey, et al., 1993; Babor, et al., 2010). Physical availability is only one of many factors; for example, although cryptomarkets democratise access to all drug types, social networks may endorse certain drugs over others. The extent to which 
people will begin to use new types of drugs depends on their attitudes towards those drugs.

For example, heroin was suddenly available but not everyone tried it as many were convinced it would be harmful for them. Our data demonstrated that there were changes to social availability among new cryptomarket users, especially for the drug users in our sample who consumed drugs alone but were (mainly) socially integrated into the online drug user forums that form part of the cryptomarket digital infrastructure. There are implications of these practices for the cultural processes of normalisation: as the social reference group people identify with changes, information and supply flows also change, and new drugs and drug practices become both more physically, socially and psychologically available. Conversely for those who took drugs within established in-person social networks, economic availability increased, as well-connected users bought in bulk and sold to friends, enabling them to receive their drugs for free or alongside financial gain ('social supply' or 'minimally commercial supply': Coomber \& Moyle, 2014).

After Silk Road closed, participants continued to access drugs through a variety of cryptomarkets and through direct purchases with dark web dealers, albeit while employing risk reduction strategies, including the use of public WIFI and using only domestic vendors. This period was also characterised by a reduction in trust of the cryptomarket environment (see Maddox et al., 2016; and also Tzanetakis, Kamphausen, Werse, \& von Laufenberg, this volume, for more detailed analysis into the development of trust within cryptomarkets). The closure of Silk Road did, however, cause disruption to established supply relationships, and changed the sense of reliable drug availability that comes from 'living on top of a bakery'. For those who wanted to avoid drug hoarding, this disruption appeared to make it more difficult for them to control their use and subsequent potential for harm from using more than intended.

\section{Limitations}

Our study design does not allow us to ascertain causal relationships between cryptomarket use and drug use trajectories. For example, a third factor, such as the type of person more likely to use cryptomarkets, may be a more important factor than cryptomarket use itself in determining the drug and harm trajectories described here. The extent to which these findings can be generalised to cryptomarket drug buyers more broadly is unknown, as the sample we attained is biased towards technically competent community participants who were prepared to spend hours chatting with a researcher about their drug use for no financial compensation. 
Nevertheless, online chat as a communication method was normal within the dark web cultural context, and the use of encryption was also normalised and encouraged. While online chat appeared an appropriate interview medium for this group, the extent to which it elicits rich and detailed accounts is contested (Barratt, 2012b; Davis, Bolding, Hart, Sherr, \& Elford, 2004), and because we could not retain contact details, we were unable to follow up participants to add further details to their accounts. The sample is also biased towards males, as no respondent identified as female. While this limits gender-based analyses, it also reflects the dark net being a male-dominated environment (see Barratt \& Maddox, Fortheoming In press). Indeed, the age and gender of our sample basically is not inconsistent with matches the demographic characteristics of 3,794 survey respondents who reported obtaining drugs from cryptomarkets (Median age 22 years, 82\% male; Barratt, Ferris, \& Winstock, 2016). There are always limitations associated with self-report data. We used member checking and our ethnographic presence and fieldwork to ascertain the validity of general statements (see Barratt \& Maddox, Fortheoming In press), but we cannot verify claims made by anonymous participants about their drug use. We acknowledge that in the context of these co-constructed interview spaces, accounts of harms and benefits are embedded in wider discourses that prompt participants to justify their behaviours rather than simply report them (Martin \& Stenner, 2004; Rødner, 2005). The drug and harm trajectories described here are best seen as examples of the different pathways where a sudden high availability of drugs may trigger various responses. Quantitative research could further investigate the prevalence of these various pathways among different populations of cryptomarket users.

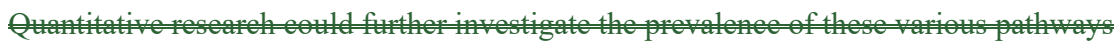
ameng different populations of eryptomarket users. A further limitation of this study is that due to the volatility and fast-moving pace of the cryptomarket ecosystem, both the findings and the methodology described here from the 2013-14 period may prove obsolete in 2016 and beyond. The environment and community feel of Silk Road does not appear to have carried through to newer cryptomarkets (Andersen \& Demant, this volume), and after multiple law enforcement operations, cryptomarket members may be even more suspicious of outsiders, making digital ethnography more difficult to conduct. Community engagement requires finding, gaining and retaining access, and such activities may become more difficult as cryptomarkets respond to threats of infiltration by law enforcement. Nevertheless, we argue that an actively-engaged digital ethnography in the cryptomarket setting is still feasible if researchers (with existing track records illustrating a commitment to conducting and 
disseminating research respectful to drug-user concerns and interests) spend a long and patient period of trust-building with these understandably suspicious communities (Barratt \& Maddox, In press).

\section{Implications $\quad$ Conclusions and Future Directions}

There are pathways through which the higher availability of drugs offered by cryptomarkets may facilitate either increased harm or reduced harm, through changes in physical, social and economic availability and the way in which 'availability' is interpreted and constructed by users. Thus it should not be assumed that the use of cryptomarkets necessarily results in increased harm, nor can it be assumed that high drug availability necessarily results in increased harm. Our study indicates that disruptions to cryptomarkets have the potential to cause unintended harm by reducing market stability and thus reducing control mechanisms used by some drug buyers as a means of 'portion control'. It is also the case that such disruptions have not deterred the buyers accessed through this study from buying drugs through the dark web, although they reported doing so with more caution. Our study cannot comment on the extent to which cryptomarket disruption has reduced drug-related harm by other mechanisms, for example, by deterring new market entrants. Given the limitations of our study, additional research is needed to help assess the extent to which policies aimed at system disruption are causing net public-health benefit or harm. Future research should also consider the implications of differential access to the opportunities and consequences arising from participation in drug cryptomarkets and the public health implications of cryptomarket diffusion into in-person 'street-level' illicit drug markets.

Cryptomarkets do appear to support a specific practice of using drugs alone (asocial use)solitary drug use while embedded within online social networks, a use pattern associated with drug-use stigma, personal preference for introspective drug experiences, and the technoelite culture. A key harm reduction message is to not use alone, and so this finding is concerning in the event of overdose, where they may not receive the necessary emergency attention. We also documented the phenomenon of cryptomarkets facilitating the initiation of illicit drug use and the resumption of use after many years of abstinence, although these examples formed the minority of the sample. Further exploration of the extent and nature of asocial solitary drug use supported by online social networks and the initiation or resumption of drug use through cryptomarkets should be conducted through surveying large samples, as 
it is unclear how representative these findings might be.

In this volatile environment, where digital social ecosystems can disappear overnight, the reliable provision of harm reduction information can be challenging. It is encouraging that anonymous digital spaces can provide a place of refuge for people who use drugs to access like-minded others (see also Barratt, 2011), but these findings also point to the need to reduce societal stigma against drug use and drug users, to enable open exchanges of information that may result in increased safety and reduced shame. Ultimately, the technological mediation of drug access, while changing some of the dynamics of the social battles for and against drug use, does not of itself change the need for open conversation within society about what drug use means and how it can be managed.

\section{Acknowledgements}

We express heartfelt gratitude to the 17 Silk Road participants who shared their stories with us, and to the various community members who engaged with us and supported our work.

We thank The Hub and the Dark Net Market Sub-Reddit for facilitating our work. We welcome your continued feedback on our ongoing work in this space. The project was supported by internal funding from the National Drug Research Institute. The National Drug Research Institute in the Faculty of Health Sciences at Curtin University and the National Drug and Alcohol Research Centre at UNSW Australia are supported by funding from the Australian Government under the Substance Misuse Prevention and Service Improvement Grants Fund. Analysis and write-up by M.B. was facilitated via a National Health \& Medical Research Council Early Career Researcher Fellowship (APP1070140), administered through UNSW Australia. M.B. gratefully acknowledges the contribution to this work of the Victorian Operational Infrastructure Support Program received by the Burnet Institute. The funders played no further part in the research process, and the views expressed in this paper should not be seen as representative of the views of the funders.

\section{References}

Abbey, A., Scott, R. O., \& Smith, M. J. (1993). Physical, subjective, and social availability: their relationship to alcohol consumption in rural and urban areas. Addiction, 88(4), 489-499.

Agar, M. H. (2006). An ethnography by any other name. Forum: Qualitative Social Research, 7(4), Article 36.

Aldridge, J., \& Décary-Hétu, D. (2014). Not an 'Ebay for Drugs': The cryptomarket 'Silk Road' as a paradigm shifting criminal innovation (May 13, 2014). SSRN. http://ssrn.com/abstract=2436643 
Andersen, R. M., \& Demant, J. (2016). Mixing politics and crime - the prevalence and decline of political discourse on the cryptomarket. International Journal of Drug Policy, This Volume.

Babor, T., Caulkins, J., Edwards, G., Fischer, B., Foxcroft, D., Humphreys, K., Obot, I., Rehm, J., Reuter, P., Room, R., Rossow, I., \& Strang, J. (2010). Drug policy and the public good. Oxford: Oxford University Press.

Barratt, M. J. (2011). Discussing illicit drugs in public internet forums: Visibility, stigma, and pseudonymity. In J. Kjeldskov \& J. Paay (Eds.), C\&T '11. Proceedings of the Fifth International Conference on Communities and Technologies, Brisbane, Australia (pp. 159-168). New York, NY: ACM.

Barratt, M. J. (2012a). Silk Road: eBay for drugs [letter to the editor]. Addiction, 107, 683.

Barratt, M. J. (2012b). The efficacy of interviewing young drug users through online chat. Drug and Alcohol Review, 31, 566-572.

Barratt, M. J., Ferris, J. A., \& Winstock, A. R. (2014). Use of Silk Road, the online drug marketplace, in the UK, Australia and the USA. Addiction, 109(5), 774-783.

Barratt, M. J., Ferris, J. A., \& Winstock, A. R. (2016). Safer scoring? Cryptomarkets, social supply and drug market violence. International Journal of Drug Policy, Forthcoming This Volume

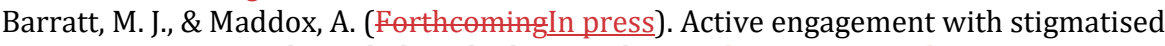
communities through digital ethnography. Qualitative Research

Christin, N. (2013, May 13-17). Traveling the Silk Road: A measurement analysis of a large anonymous online marketplace. Paper presented at the International World Wide Web Conference (IW3C2), Rio de Janeiro, Brazil.

Coomber, R., \& Moyle, L. (2014). Beyond drug dealing: Developing and extending the concept of 'social supply' of illicit drugs to 'minimally commercial supply'. Drugs: Education, Prevention, and Policy, 21, 157-164.

Davis, M., Bolding, G., Hart, G., Sherr, L., \& Elford, J. (2004). Reflecting on the experience of interviewing online: Perspectives from the Internet and HIV study in London. AIDS Care, 16, 944-952.

Décary-Hétu, D., \& Aldridge, J. (2015). Sifting through the net: Monitoring of online offenders by researchers. European Review of Organised Crime, 2, 122-141.

Dingledine, R., Mathewson, N., \& Syverson, P. (2004, August 9-13). Tor: the secondgeneration onion router. Paper presented at the SSYM'04 Proceedings of the 13th conference on USENIX Security Symposium, San Diego, CA, USA.

Dolliver, D. S. (2015). Evaluating Drug Trafficking on the Tor Network: Silk Road 2, the Sequel. International Journal of Drug Policy, 26, 1113-1123.

Falzon, M.-A. (2009). Introduction. In M.-A. Falzon (Ed.), Multi-sited ethnography: Theory, praxis and locality in contemporary research (pp. 1-23). Surrey, England: Ashgate.

Freisthler, B., \& Gruenewald, P. J. (2014). Examining the relationship between the physical availability of medical marijuana and marijuana use across fifty California cities. Drug and Alcohol Dependence, 143, 244-250.

Gmel, G., Holmes, J., \& Studer, J. (2015). Are alcohol outlet densities strongly associated with alcohol-related outcomes? A critical review of recent evidence. Drug and Alcohol Review, Early View. doi: 10.1111/dar.12304

Gruenewald, P. (2008). Why do alcohol outlets matter anyway? A look into the future. Addiction, 103, 1585-1587.

Hine, C. (2008). Virtual ethnography: Modes, varieties, affordances. In N. Fielding, R. M. Lee \& G. Blank (Eds.), The Sage handbook of online research methods (pp. 257- 
270). London, England: Sage.

Kerr, T., Small, W., \& Wood, E. (2005). The public health and social impacts of drug market enforcement: A review of the evidence. International Journal of Drug Policy, 16, 210-220.

Liang, W., \& Chikritzhs, T. (2011). Revealing the link between licensed outlets and violence: Counting venues versus measuring alcohol availability. Drug and Alcohol Review, 30, 524-535.

Livingston, M. (2008). A longitudinal analysis of alcohol outlet density and assault. Alcoholism: Clinical and Experimental Research, 32, 1074-1079.

Livingston, M. (2013). Commentary on Wang et al. (2013): Time to test the mechanisms of alcohol outlet density effects. Addiction, 108, 2089-2090.

Livingston, M. (2014). Alcohol outlet density: The challenge of linking research findings to policy. Australasian Epidemiologist, 21(2), S22-S24.

Maddox, A., Barratt, M. J., Allen, M., \& Lenton, S. (2016). Constructive activism in the dark web: Cryptomarkets and illicit drugs in the digital 'demimonde'. Information, Communication and Society, 19, 111-126.

Martin, A., \& Stenner, P. (2004). Talking about drug use: What are we (and our participants) doing in qualitative research? International Journal of Drug Policy, $15,395-405$.

Martin, J. (2014). Drugs on the dark net: how cryptomarkets are transforming the global trade in illicit drugs. New York: Palgrave Pivot.

McHugh, M. L. (2012). Interrater reliability: The kappa statistic. Biochemia Medica, 22, 276-282.

Phelps, A., \& Watt, A. (2014). I shop online - recreationally! Internet anonymity and Silk Road enabling drug use in Australia. Digital Investigation, 11, 261-272.

Rødner, S. (2005). "I am not a drug abuser, I am a drug user": A discourse analysis of 44 drug users' construction of identity. Addiction Research and Theory, 13, 333-346.

Sanders, B., Lankenau, S. E., Bloom, J. J., \& Hathazi, D. (2008). "Research chemicals": Tryptamine and phenethylamine use among high-risk youth. Substance Use and Misuse, 43, 389-402.

Shanahan, M., \& Ritter, A. (2014). Cost benefit analysis of two policy options for cannabis: Status quo and legalisation. PLoS ONE, 9(4), e95569.

Stockwell, T., \& Gruenewald, P. J. (2004). Controls on the physical availability of alcohol. In N. Heather \& T. Stockwell (Eds.), The Essential Handbook of Treatment and Prevention of Alcohol Problems (pp. 213-234). New York: Wiley.

Tzanetakis, M., Kamphausen, G., Werse, B., \& von Laufenberg, R. (2016). The transparency paradox. Building trust, resolving disputes and optimising logistics on conventional and online drugs markets. International Journal of Drug Policy, This Volumedoi: 10.1016/j.drugpo.2015.12.010

van Hout, M. C., \& Bingham, T. (2013a). 'Silk Road', the virtual drug marketplace: A single case study of user experiences. International Journal of Drug Policy, 24, 385-391.

van Hout, M. C., \& Bingham, T. (2013b). 'Surfing the Silk Road': A study of users' experiences. International Journal of Drug Policy, 24, 524-529.

van Hout, M. C., \& Bingham, T. (2014). Responsible vendors, intelligent consumers: Silk Road, the online revolution in drug trading. International Journal of Drug Policy, 25, 183-189. 